\title{
Cosmic Bell Test Using Random Measurement Settings from High-Redshift Quasars
}

\author{
Dominik Rauch, ${ }^{1,2, *}$ Johannes Handsteiner, ${ }^{1,2}$ Armin Hochrainer, ${ }^{1,2}$ Jason Gallicchio, ${ }^{3}$ Andrew S. Friedman, ${ }^{4}$ \\ Calvin Leung, ${ }^{1,2,3,5}$ Bo Liu, ${ }^{6}$ Lukas Bulla, ${ }^{1,2}$ Sebastian Ecker, ${ }^{1,2}$ Fabian Steinlechner, ${ }^{1,2}$ Rupert Ursin, ${ }^{1,2}$ \\ Beili Hu, ${ }^{3}$ David Leon, ${ }^{4}$ Chris Benn, ${ }^{7}$ Adriano Ghedina, ${ }^{8}$ Massimo Cecconi ${ }^{8}$ Alan H. Guth, ${ }^{5}$ \\ David I. Kaiser, ${ }^{5, \dagger}$ Thomas Scheidl, ${ }^{1,2}$ and Anton Zeilinger ${ }^{1,2, \$}$ \\ ${ }^{1}$ Institute for Quantum Optics and Quantum Information (IQOQI), Austrian Academy of Sciences, \\ Boltzmanngasse 3, 1090 Vienna, Austria \\ ${ }^{2}$ Vienna Center for Quantum Science \& Technology (VCQ), Faculty of Physics, University of Vienna, \\ Boltzmanngasse 5, 1090 Vienna, Austria \\ ${ }^{3}$ Department of Physics, Harvey Mudd College, Claremont, California 91711, USA \\ ${ }^{4}$ Center for Astrophysics and Space Sciences, University of California, San Diego, La Jolla, California 92093, USA \\ ${ }^{5}$ Department of Physics, Massachusetts Institute of Technology, Cambridge, Massachusetts 02139, USA \\ ${ }^{6}$ School of Computer, NUDT, 410073 Changsha, China \\ ${ }^{7}$ Isaac Newton Group, Apartado 321, 38700 Santa Cruz de La Palma, Spain \\ ${ }^{8}$ Fundación Galileo Galilei-INAF, 38712 Breña Baja, Spain
}

(Received 5 April 2018; revised manuscript received 14 June 2018; published 20 August 2018)

\begin{abstract}
In this Letter, we present a cosmic Bell experiment with polarization-entangled photons, in which measurement settings were determined based on real-time measurements of the wavelength of photons from high-redshift quasars, whose light was emitted billions of years ago; the experiment simultaneously ensures locality. Assuming fair sampling for all detected photons and that the wavelength of the quasar photons had not been selectively altered or previewed between emission and detection, we observe statistically significant violation of Bell's inequality by 9.3 standard deviations, corresponding to an estimated $p$ value of $\lesssim 7.4 \times 10^{-21}$. This experiment pushes back to at least $\sim 7.8$ Gyr ago the most recent time by which any local-realist influences could have exploited the "freedom-of-choice" loophole to engineer the observed Bell violation, excluding any such mechanism from $96 \%$ of the space-time volume of the past light cone of our experiment, extending from the big bang to today.
\end{abstract}

DOI: 10.1103/PhysRevLett.121.080403

Background.-To Erwin Schrödinger, entanglement was "the characteristic trait of quantum mechanics, the one that enforces its entire departure from classical lines of thought" [1]. He referred to an analysis by Einstein, Podolsky, and Rosen (EPR) [2], regarding the quantum-mechanical predictions for perfect correlations in certain quantum systems. EPR made two assumptions explicit. Regarding locality, they wrote: "Since at the time of measurement the two systems no longer interact, no real change can take place in the second system in consequence of anything that may be done to the first system." They also articulated a "reality criterion": "If, without in any way disturbing a system, we can predict with certainty (i.e., with probability equal to unity) the value of a physical quantity, there exists an element of physical reality corresponding to this physical quantity." In the light of these two assumptions and their

Published by the American Physical Society under the terms of the Creative Commons Attribution 4.0 International license. Further distribution of this work must maintain attribution to the author(s) and the published article's title, journal citation, and DOI. analysis of a particular two-particle state, EPR concluded that quantum mechanics is incomplete. While the EPR reasoning is logically unassailable, Niels Bohr pointed out that the EPR assumptions need not hold for quantum observations [3].

This discussion had laid dormant for several decades, but in 1964, John Stewart Bell demonstrated that a complete theory based on the EPR premises makes predictions that are in conflict with those of quantum mechanics $[4,5]$. In such local-realist theories, it is assumed that every individual system carries its own set of properties prior to measurement, which are presumed to be independent of any possible influence from outside its past light cone. Bell concluded that in a local-realist theory the strength of correlations among measurements on different particles' properties is limited and smaller than the predictions of quantum physics. This is expressed by Bell's inequality.

With Bell's result, a question that previously had been dismissed as "merely philosophical" became experimentally testable. In 1969, Clauser, Horne, Shimony, and Holt (CHSH) published their inequality as an experimentally accessible variant of Bell's version [6]. The idea was to measure the four probabilities $p\left(A, B \mid a_{i}, b_{j}\right)$ of 
measurement results $A, B \in\{+1,-1\}$, in which Alice chooses between two measurement bases $a_{1}$ and $a_{2}$, and likewise Bob chooses between the two measurement bases $b_{1}$ and $b_{2}$. For systems in particular states subject to judicious choices of measurement bases, the predictions for correlations among the measurement outcomes $A, B$ under various combinations of settings $a_{i}, b_{j}$ differ markedly between quantum mechanics and models that satisfy EPR's assumptions of locality and realism.

Subsequently, entangled-particle states have been shown to violate Bell's inequality in numerous situations, consistent with the predictions of quantum theory [7-9]. Yet experiments always require sets of assumptions for their interpretation [10,11]. In tests of local realism, these assumptions can be seen as loopholes, by which, in principle, it could be argued that local realism has not been completely ruled out $[8,12]$. Closing the locality loophole $[13,14]$, for example, requires that the measurement settings are changed by Alice shortly before the arrival of an entangled particle at her detector, such that no signal could inform Bob about Alice's measurement setting or outcome before Bob completes a measurement at his own detector (and vice versa). The fair sampling assumption, on the other hand, states that the measured subset of particles is representative of the complete set. This loophole is closed if a sufficiently high fraction of the entangled pairs is detected [15-17]. Recently, several experiments have observed significant violations of Bell's inequality while simultaneously closing both the locality and fair-sampling loopholes [18-21].

Arguably the most interesting assumption is that the choice of measurement settings is "free and random," and independent of any physical process that could affect the measurement outcomes [5,22-25]. As Bell himself noted, his inequality was derived under the assumption "that the settings of instruments are in some sense free variablessay at the whim of experimenters-or in any case not determined in the overlap of the backward light cones" [22]. In recent years, this "freedom-of-choice" loophole has garnered significant theoretical interest [26-35], as well as growing experimental attention [36-41].

The freedom-of-choice loophole, as usually understood, concerns events that might have transpired within the causal past of a given experiment, which a local-realist mechanism could have exploited in order to mimic the predictions from quantum mechanics [42]. In a recent pilot test [38], measurement settings for a test of Bell's inequality were determined by real-time observation of light from Milky Way stars, thereby constraining any such localrealist mechanism to have acted no more recently than $\sim 600$ years ago, rather than microseconds before a given experimental run (as in previous tests [36]). The magnitude of that leap reflected how comparatively little attention had been devoted previously to experimentally addressing this loophole. Given the expansion history of the Universe since the big bang, however, the pilot test [38] excluded only about one hundred-thousandth of one percent of the relevant spacetime volume within the past light cone of the experiment.

In this Letter, we describe a cosmic Bell experiment that pushes the origin of the measurement settings considerably deeper into cosmic history, constraining any local-realist mechanism to have acted no more recently than $7.78 \mathrm{Gyr}$ ago. Based on the arrangement of high-redshift quasars used in our experiment, these results exclude any localrealist mechanism that might have exploited the freedomof-choice loophole from $96.0 \%$ of the space-time volume of the past light cone of the experiment, extending from the big bang to today.

Experimental implementation.-Figure 1 shows a schematic of the experimental setup at the Observatorio del Roque de los Muchachos on the Canary Island of La Palma. A central entangled photon source was located in a container next to the Nordic Optical Telescope. One entangled-photon observer, Alice, was situated in another container next to the Telescopio Nazionale Galileo (TNG), and Bob was stationed at the ground floor of the William Herschel Telescope (WHT). The quasar photons were collected by the TNG [46] and the WHT [47]. The random numbers extracted from these signals were transmitted to the observers using BNC cables. The polarizationentangled photons were distributed from the source to the receivers via free-space optical links. A more detailed schematic of the setup can be seen in Fig. 2.

The entangled photon source (see Supplemental Material [48]) was based on type 0 spontaneous parametric downconversion (SPDC) in a Sagnac loop configuration [73,74]. It generated fiber-coupled photon pairs at center wavelengths $\lambda_{\mathcal{A}}=850 \mathrm{~nm}$ and $\lambda_{\mathcal{B}}=773.6 \mathrm{~nm}$ in a state close to the maximally entangled Bell state $\left|\Phi^{ \pm}\right\rangle=(1 / \sqrt{2})\left(\left|H_{\mathcal{A}} H_{\mathcal{B}}\right\rangle \pm\right.$ $\left.\left|V_{\mathcal{A}} V_{\mathcal{B}}\right\rangle\right)$, where subscripts $\mathcal{A}$ and $\mathcal{B}$ label the respective single-mode fiber for Alice and Bob. Each photon was guided to a transmitting telescope (Tx) and distributed via free-space optical channels to the receiving stations of Alice and Bob. Each station consisted of a receiving telescope for entangled photons (Rx), a polarization analyzer (POL), a control and data acquisition unit $(\mathrm{CaDa})$ locked to a rubidium frequency standard, and a cosmic random number generator (CRNG). The entangled photons were guided from the Rx to the polarization analyzer, where an electro-optical modulator (EOM) performed the fast switching between the complementary measurement bases accordingly. The polarization was measured using a polarizing beam splitter followed by a single-photon avalanche diode (SPAD) in each output port.

The CRNGs at TNG (Alice) and WHT (Bob) were essentially identical. The optical path for each CRNG featured a magnified intermediate image, which enabled one to adjust the field of view with an iris in order to minimize background light. A dichroic mirror with a cutoff wavelength of $630 \mathrm{~nm}$ split the incoming light in a transmitted "red" and a reflected "blue" arm. Additional 


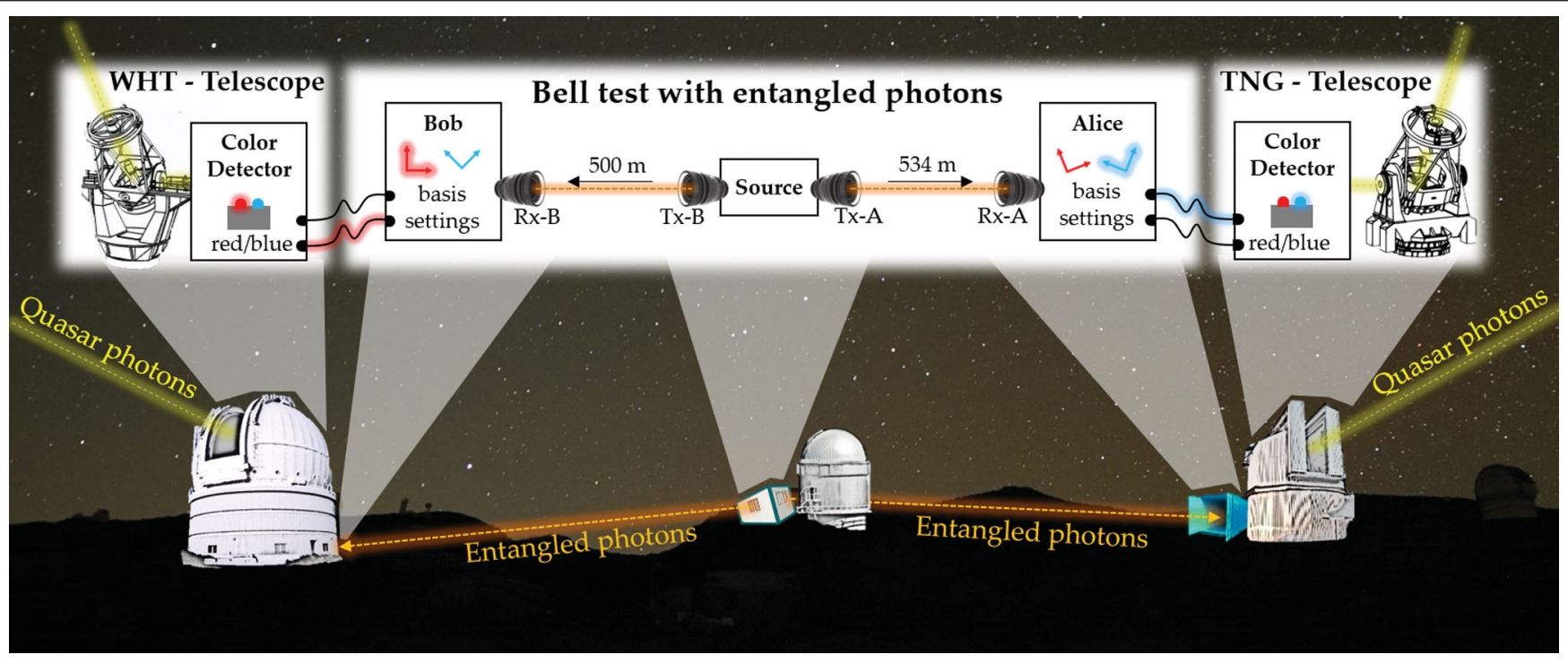

FIG. 1. The experimental stations for our cosmic Bell test. Alice's station received cosmic photons with the Telescopio Nazionale Galileo (TNG), whose primary mirror diameter is $3.58 \mathrm{~m}$, while Bob's station received cosmic photons with the William Herschel Telescope (WHT), whose primary mirror diameter is $4.20 \mathrm{~m}$. Polarization entangled photons were sent from the source to Alice and Bob. Diameters and focal lengths of the quantum channel telescopes were Tx: $d=70 \mathrm{~mm}, f=280 \mathrm{~mm}$; Rx: $d=140 \mathrm{~mm}$, $f_{\text {eff }}=1470 \mathrm{~mm}$. Latitude, longitude, and elevation for the experimental sites were Alice $\left(\mathcal{A}: 28.75410^{\circ},-17.88915^{\circ}, 2375 \mathrm{~m}\right)$, $\operatorname{Bob}\left(\mathcal{B}: 28.760636^{\circ},-17.8816861^{\circ}, 2352 \mathrm{~m}\right)$, and the Source $\left(\mathcal{S}: 28.757189^{\circ},-17.884961^{\circ}, 2385 \mathrm{~m}\right)$. The distances from Source to Bob and Alice were 500 and $534 \mathrm{~m}$, respectively.

filters (shortpass at $620 \mathrm{~nm}$ in the blue arm and longpass at $637 \mathrm{~nm}$ in the red arm) efficiently filtered out misdirected photons whose wavelengths were near the cutoff wavelength of the dichroic mirror. Incorporating these additional filters yielded much smaller fractions of misdirected astronomical photons than in our previous experiment [38], with $f_{w}<2 \times 10^{-5}$ (see the Supplemental Material [48]). Light from each arm was fed to a SPAD. Electric signals from the SPADs were processed by the $\mathrm{CaDa}$, which triggered the EOM to apply the corresponding

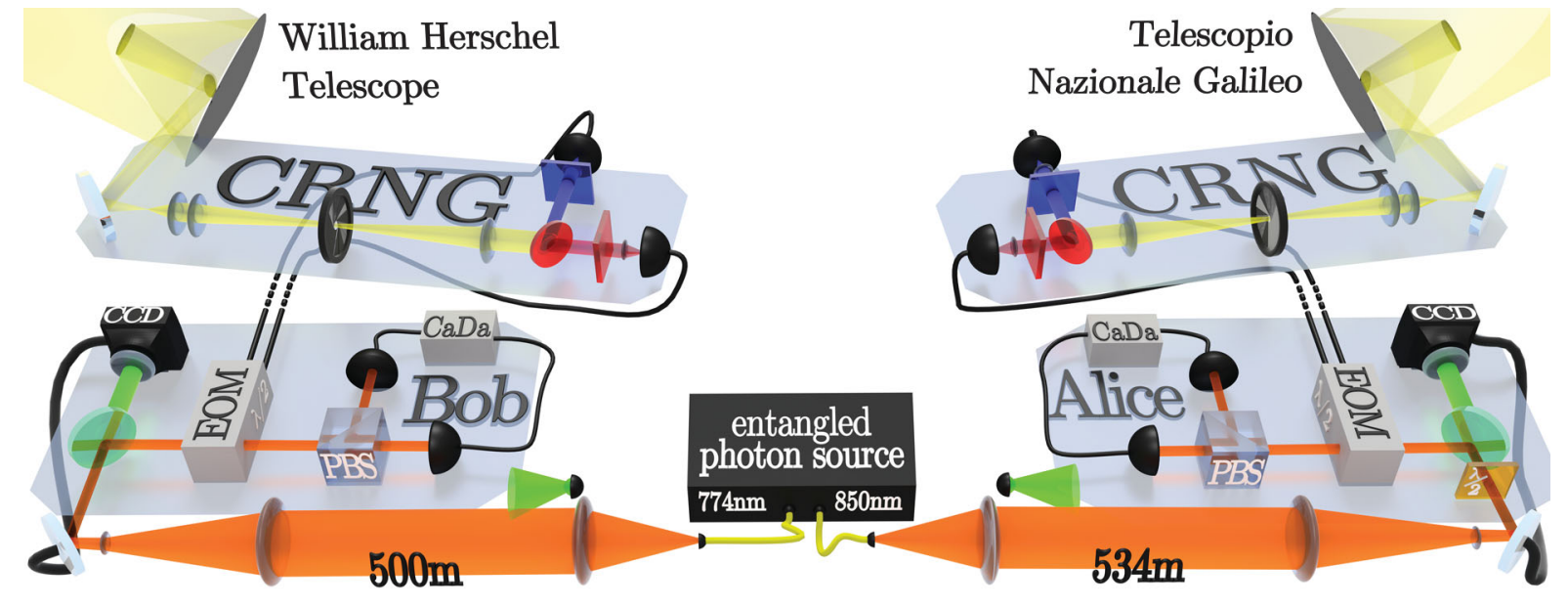

FIG. 2. A photon pair source located in the middle produced polarization-entangled photons at center wavelengths of 773.6 and $850 \mathrm{~nm}$. The photons were separated into two spatial modes via a dichroic mirror and sent via free-space channels to the quantum receivers at Bob $(773.6 \mathrm{~nm})$ and Alice $(850 \mathrm{~nm})$. Fast steering mirrors guided the photons to the receivers using a green LED as a reference. Electro-optical modulators (EOM) rotate the measurement basis according to the input signals from the CRNGs. Polarization measurements are performed using a polarizing beam splitter (PBS) with avalanche photodiodes in each output path. Detection events are time stamped by the control and data acquisition unit $(\mathrm{CaDa})$ and stored locally. Quasar light is collected by the astronomical telescopes and fed into an optical system that creates a magnified image with an iris to restrict the field of view. The quasar light is then split according to its wavelength into a "blue" and a "red" channel, whereby each channel contains additional filters to remove misdirected photons. The detector signals are used to trigger the implementation of the corresponding measurement basis at the EOM. 
measurement settings. Alice measured linear polarization along $22.5^{\circ} / 112.5^{\circ}$ (red) and $67.5^{\circ} / 157.5^{\circ}$ (blue), while Bob measured linear polarization along $0^{\circ} / 90^{\circ}$ (red) and $45^{\circ} / 135^{\circ}$ (blue). All SPAD events, from the CRNGs and the polarization analyzers, were time stamped in the $\mathrm{CaDa}$ and recorded by a computer.

Using the wavelength of cosmic photons to implement the measurement settings requires the assumption that the wavelength of each photon was set at emission and has not been selectively altered or previewed between emission and detection. (Well-known processes, such as cosmological redshift and gravitational lensing, treat all photons from a given astronomical source in a uniform way, independent of the photons' wavelength at emission [75-77]).

Within an optically linear medium, there does not exist any known physical process that can absorb and reradiate a given photon at a different wavelength along the same line of sight, without violating the local conservation of energy and momentum. We further assume that the detected cosmic photons represent a fair sample, despite significant losses in the intergalactic and interstellar media, the Earth's atmosphere, and the experimental setup.

Various scenarios could (in principle) lead to corrupt choices of measurement settings within our experiment. For example, local sources of photons ("noise") rather than genuine astronomical photons could trigger the CRNGs. The most significant sources of local noise include sky glow, light pollution, and detector dark counts. The overall background was measured by pointing each telescope to a dark sky patch 10 arcseconds away from its target quasar before and after each observing period.

Space-time arrangement.-Ensuring locality requires that any information leaving Alice's quasar at the speed of light along with her setting-determining cosmic photon could not have reached Bob before his measurement of the entangled photon is completed and vice versa.

The projected space-time diagram in Fig. 3 illustrates the situation for the first observed quasar pair (pair 1) of our experiment. The entangled photons are generated at point $\mathcal{S}$ and travel through $12 \mathrm{~m}$ of optical fiber, resulting in a delay of $\tau_{\text {fiber }} \approx 50 \mathrm{~ns}$. The distance over the free-space channels is $x_{\mathcal{A}}=534 \mathrm{~m}$ to $\mathcal{A}$ and $x_{\mathcal{B}}=500 \mathrm{~m}$ to $\mathcal{B}$.

To ensure the locality conditions, measurements of entangled photons must only be accepted within a certain valid time window $\tau_{\text {valid, }}^{k}$ which has to be chosen such that the selection and implementation of the corresponding settings on one side remain spacelike separated from the measurements on the other side. Here, $\tau_{\text {valid }}^{k}$ is constrained to within a certain time window $\tau_{\text {geom }}^{k}$, which depends on the time-dependent directions of the quasars relative to $\mathcal{A}$ and $\mathcal{B}$. Given the moderate time dependence of $\tau_{\text {geom }}^{k}$ over the relatively brief observing periods $(\leq 17 \mathrm{~min})$, we use the shortest value per side within the observing period: $\bar{\tau}_{\text {geom }}^{k}=\min _{t}\left(\tau_{\text {geom }}^{k}\right)$, where $\bar{\tau}_{\text {geom }}^{\mathcal{A}}=2.81 \mu \mathrm{s}(2.67 \mu \mathrm{s})$ and $\bar{\tau}_{\text {geom }}^{\mathcal{B}}=1.48 \mu \mathrm{s}(1.11 \mu \mathrm{s})$ for pair 1 (2). Various delays

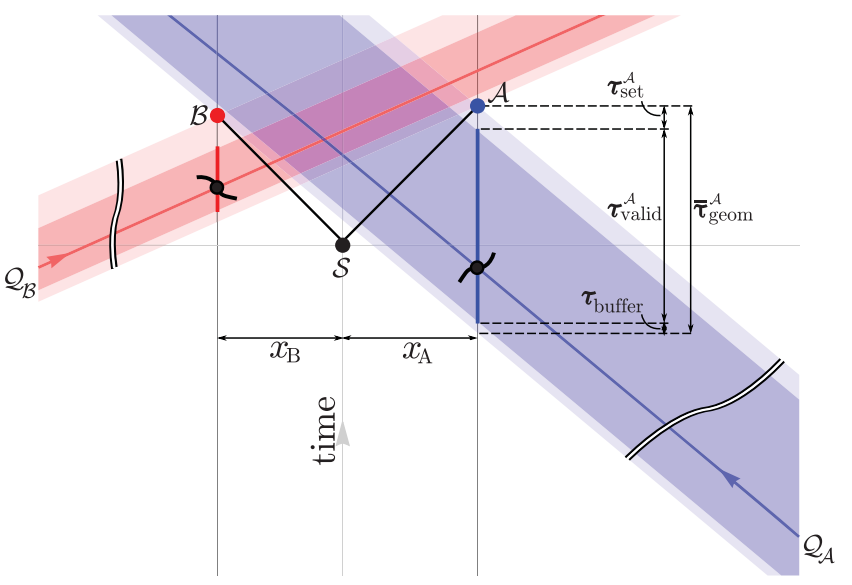

FIG. 3. $(1+1)$ D space-time diagram for pair 1, with the origin at the source $\mathcal{S}$ of entangled pair creation (black dot) and a spatial projection axis chosen to minimize its distance to Alice and Bob. After a short fiber delay (too small to see), entangled photons are sent via free-space channels (black lines) to be measured by Alice and Bob at events $A$ and $B$. Galaxy symbols indicate examples of measurements of valid settings from quasar photons emitted far away at space-time events $\mathcal{Q}_{A}$ and $\mathcal{Q}_{B}$. Ensuring locality limits settings to the shaded regions. Delays to implement each setting and an added safety buffer shorten the validity time windows that were actually used to the darker shaded regions.

from signal transmission through fibers and BNC cables, and to implement a given setting with the EOM, have to be subtracted from $\tau_{\text {geom }}^{k}$ to compute the correct validity time $\tau_{\text {valid }}^{k}$. The delay until a certain setting was implemented $\tau_{\text {set }}^{k}$ was measured to be 325 and 430 ns for Alice and Bob, respectively. An additional buffer was used on both sides with $\tau_{\text {buffer }}=150 \mathrm{~ns}$ to account for small inaccuracies in timing and distance measurements (for details please refer to the Supplemental Material [48]). The final validity time we used is then $\tau_{\text {valid }}^{k}=\bar{\tau}_{\text {geom }}^{k}-\tau_{\text {set }}^{k}-\tau_{\text {buffer }}$.

For pairs 1 and 2, measurement settings at Bob's station were determined based on observations of a quasar with redshift $z_{\mathcal{B}}=3.911$ [78], corresponding to a lookback time to the emission of that light $t_{\mathrm{lb}}^{\mathcal{B}}=12.21 \mathrm{Gyr}$ ago. Measurement settings at Alice's station were determined based on observations of quasars with $z_{\mathcal{A}}=0.964$ [79] (pair 1) and $z_{\mathcal{A}}=0.268$ [80] (pair 2), corresponding to $t_{\mathrm{lb}}^{\mathcal{A}}=7.78$ and $3.22 \mathrm{Gyr}$ ago, respectively (see Table I). These times may be compared with the age of our observable Universe since the big bang, $t_{\mathrm{lb}}=13.80 \mathrm{Gyr}$ [81]. We consider possible implications of inhomogeneities along the lines of sight to these objects, such as gravitational lensing effects, in the Supplemental Material [48].

Figure 4 depicts the past light cone of our experiment (gray) together with the past light cones of quasar emission events $\mathcal{Q}_{\mathcal{A}}$ (blue) and $\mathcal{Q}_{\mathcal{B}}$ (red) for the quasars of pair 1 . The past light cones from $\mathcal{Q}_{\mathcal{A}}$ and $\mathcal{Q}_{\mathcal{B}}$ for this pair last intersected $t_{\mathrm{lb}}^{\mathcal{A B}}=13.15 \mathrm{Gyr}$ ago, less than 650 million years after the big bang. (For pair 2, the past light cones 
TABLE I. For Alice and Bob's side $(k=\{\mathcal{A}, \mathcal{B}\})$, we list the QSO Simbad identifiers, azimuth (az ${ }_{k}$ ) (clockwise from due North) and altitude $\left(\mathrm{alt}_{k}\right)$ above horizon at the start of the observing periods, and redshift $(z)$ and lookback time to emission $\left(t_{\mathrm{lb}}\right)$ for quasars observed in pairs 1 and 2, beginning at UTC 2018-01-11 00:20:00 (pair 1) and 2018-01-11 01:21:00 (pair 2). Pair 1 was observed for a total of $17 \mathrm{~min}$, pair 2 for $12 \mathrm{~min}$. Here, $\tau_{\text {valid }}^{k}$ is the time the detector setting was valid, taking into account delays and safety margins (see Fig. 3). The last three columns show the measured CHSH parameter, as well as the $p$ value and the number of standard deviations $\nu$ by which our local-realist model can be rejected (see the Supplemental Material [48]).

\begin{tabular}{|c|c|c|c|c|c|c|c|c|c|c|}
\hline Pair & Side & ID & $a z_{\mathrm{k}}^{\circ}$ & $\mathrm{alt}_{\mathrm{k}}^{\circ}$ & $z$ & $t_{\mathrm{lb}}[\mathrm{Gyr}]$ & $\tau_{\text {valid }}^{k}[\mu \mathrm{s}]$ & $S_{\text {exp }}$ & $p$ value & $\nu$ \\
\hline \multirow[t]{2}{*}{1} & $\mathcal{A}$ & QSO B0350 - 073 & 233 & 38 & 0.964 & 7.78 & 2.34 & 2.65 & $7.4 \times 10^{-21}$ & 9.3 \\
\hline & $\mathcal{B}$ & QSO J0831 + 5245 & 35 & 57 & 3.911 & 12.21 & 0.90 & & & \\
\hline \multirow[t]{2}{*}{2} & $\mathcal{A}$ & QSO B0422 + 004 & 246 & 38 & 0.268 & 3.22 & 2.20 & 2.63 & $7.0 \times 10^{-13}$ & 7.1 \\
\hline & $\mathcal{B}$ & QSO J0831 + 5245 & 21 & 64 & 3.911 & 12.21 & 0.53 & & & \\
\hline
\end{tabular}

most recently intersected $t_{\mathrm{lb}}^{A B}=12.47 \mathrm{Gyr}$ ago.) This is the most recent time by which a correlation between the two quasars could have occurred or been orchestrated. The space-time 4-volume contained within the union of the past light cones from $\mathcal{Q}_{\mathcal{A}}$ and $\mathcal{Q}_{\mathcal{B}}$ constitutes just $4.0 \%$ (pair 1 ) and $36.5 \%$ (pair 2) of the 4-volume within the past light cone of our experiment, respectively (see Supplemental Material [48]). Events associated with any local-realist mechanism that could have affected detector settings and measurement outcomes of our experiment would need to lie within the past light cones of $\mathcal{Q}_{\mathcal{A}}$ and/or $\mathcal{Q}_{\mathcal{B}}$ and hence are

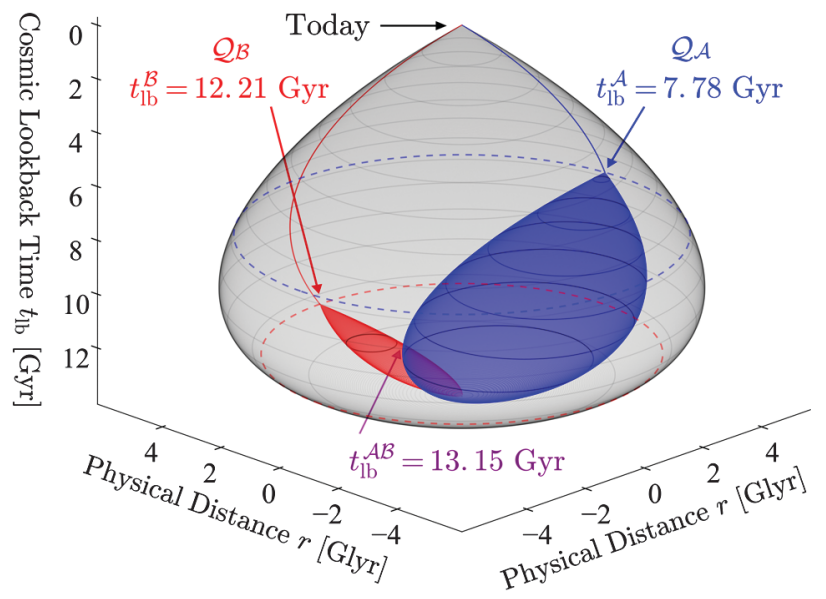

FIG. 4. $(2+1)$ D space-time diagram for pair 1, showing the past light cone of our experiment (gray) and of the quasar emission events $\mathcal{Q}_{\mathcal{A}}$ (blue) and $\mathcal{Q}_{\mathcal{B}}$ (red), extending back to the big bang, $13.80 \mathrm{Gyr}$ ago. The quasars in pair 1 emitted the light that we observed during our experiment $t_{\mathrm{lb}}^{\mathcal{A}}=7.78 \mathrm{Gyr}$ and $t_{\mathrm{lb}}^{\mathcal{B}}=$ 12.21 Gyr ago. The past light cones from $\mathcal{Q}_{\mathcal{A}}$ and $\mathcal{Q}_{\mathcal{B}}$ last intersected $t_{\mathrm{lb}}^{\mathcal{A B}}=13.15 \mathrm{Gyr}$ ago. The shapes of the light cones reflect the changing rate of cosmic expansion since the big bang. To be consistent with our data, any local-realist mechanism would need to have affected detector settings and measurement outcomes of our experiment from within the past light cones of events $\mathcal{Q}_{\mathcal{A}}, \mathcal{Q}_{\mathcal{B}}$, or their overlap, a space-time region that consists of only $4.0 \%$ of the physical space-time volume contained within the past light cone of our experiment. Such a local-realist scenario would need to have been set in motion at least 7.78 Gyr ago. restricted to have acted no more recently than $t_{\mathrm{lb}}^{\mathcal{A}}=7.78$ or 3.22 Gyr ago for pairs 1 and 2, respectively.

Analysis and results.-We performed two cosmic Bell tests with the quasars listed in Table I, for a total measurement time of $17 \mathrm{~min}$ (pair 1) and $12 \mathrm{~min}$ (pair 2 ). In the analysis of our acquired data, we follow the assumption of fair sampling and fair coincidences [12]. Thus, our data can be postselected for coincidence events at Alice's and Bob's stations. We correct for the clock drift as in Ref. [82] and identify coincidences within a time window of $2.66 \mathrm{~ns}$. We then check for correlations between measurement outcomes $A, B \in\{+1,-1\}$ for particular settings choices $\left(a_{i}, b_{j}\right), i, j \in\{1,2\}$ using the ClauserHorne-Shimony-Holt (CHSH) inequality [6]:

$$
S \equiv\left|E_{11}+E_{12}+E_{21}-E_{22}\right| \leq 2
$$

where $E_{i j}=2 p\left(A=B \mid a_{i} b_{j}\right)-1$ and $p\left(A=B \mid a_{i} b_{j}\right)$ is the probability of Alice and Bob obtaining the same measurement outcome for the joint settings $\left(a_{i}, b_{j}\right)$. While four probabilities can arithmetically add up to 4 , local-realistic correlations cannot exceed an $S$ value of 2 , and the quantum-mechanical limit is $2 \sqrt{2}$ [83].

As can be seen from Table I, the measured $S_{\exp }$ values are 2.65 and 2.63 for pairs 1 and 2, respectively, which clearly exceed the local-realist bound of 2 . However, not all of our settings were determined by genuine cosmic photons. A certain fraction of settings $\epsilon_{k}$ on each side $(k \in\{\mathcal{A}, \mathcal{B}\})$ was produced by some kind of local process, including sky glow, ambient light, and detector dark counts. We therefore consider such settings to be "corrupt" and assume that a local-realist mechanism could have exploited them to produce maximal CHSH correlations, with $S=4$. Such a (hypothetical) mechanism could produce $\mathrm{CHSH}$ correlations as large as $S=2\left(1-\epsilon_{\mathcal{A}}-\epsilon_{\mathcal{B}}\right)+4\left(\epsilon_{\mathcal{A}}+\epsilon_{\mathcal{B}}\right)$ [38,40,84].

In our analysis, we account for such "corrupt" settings as well as unequal (biased) frequencies for various combinations of detector settings $\left(a_{i}, b_{j}\right)$ and possible "memory effects" by which a local-realist mechanism could exploit knowledge of settings and outcomes of previous trials (see the Supplemental Material [48]). From this detailed 
treatment, we find that correlations at least as large as observed in our data could have been produced by a localrealist mechanism only with probabilities $p \leq 7.4 \times 10^{-21}$ for pair 1 and $p \leq 7.0 \times 10^{-13}$ for pair 2, corresponding to experimental violations of the Bell-CHSH bound by at least 9.3 and 7.1 standard deviations, respectively.

Conclusions.-For each cosmic Bell test reported here, we assume fair sampling and close the locality loophole. We also constrain the freedom-of-choice loophole with detector settings determined by extragalactic events, such that any local-realist mechanism would need to have acted no more recently than 7.78 or 3.22 Gyr ago for pairs 1 and 2 , respectively-more than six orders of magnitude deeper into cosmic history than the experiments reported in Ref. [38]. This corresponds to excluding such local-realist mechanisms from $96.0 \%$ (pair 1) and $63.5 \%$ (pair 2) of the relevant space-time regions, compared to $\sim 10^{-5} \%$ of the relevant space-time region as in Ref. [38] (see the Supplemental Material [48]).

We have therefore dramatically limited the space-time regions from which local-realist mechanisms could have affected the outcome of our experiment to early in the history of our Universe. To constrain such models further, one could use other physical signals to set detector settings, such as patches of the cosmic microwave background radiation $(\mathrm{CMB})$ or even primordial neutrinos or gravitational waves, thereby constraining such models all the way back to the big bang-or perhaps even earlier, into a phase of early-Universe inflation [31,38]. Such extreme tests might ultimately prove relevant to the question of whether quantum entanglement undergirds the emergence of spacetime itself (for a recent review, see Ref. [85]).

The authors would like to thank Cecilia Fariña, Émilie L'Homé, Karl Kolle, Neil O’Mahony, Jürg Rey, Fiona Riddick, and the whole team at the WHT as well as Emilio Molinari, Giovanni Mainella, Carlos Gonzalez, and the whole team at the TNG for their tremendous support of our experiment. We also thank Thomas Augusteijn, Carlos Perez, and all the staff at the NOT for their support, which did not decrease even after our container crashed into their telescope in a storm. We are also grateful for the encouraging support of Cesare Barbieri. In addition, we are grateful to Brian Keating, Hien Nguyen, Paul Schechter, and Gary Cole for helpful discussions. This work was supported by the Austrian Academy of Sciences (OEAW), by the Austrian Science Fund (FWF) with SFB F40 (FOQUS) and FWF project CoQuS No. W1210-N16, the Austrian Federal Ministry of Education, Science and Research (BMBWF), and the University of Vienna via the project QUESS. This work was also supported by NSF INSPIRE Grant No. PHY-1541160. Portions of this work were conducted in MIT's Center for Theoretical Physics and supported in part by the U.S. Department of Energy under Contract No. DE-SC0012567. C. L. was supported by the U.S. Department of Defense (DoD) through the
National Defense Science \& Engineering Graduate Fellowship (NDSEG) Program.

Note added.-After we completed our experiment, a similar experiment was conducted by another group, the results of which are reported in Ref. [86].

*dominik.rauch@oeaw.ac.at

†dikaiser@mit.edu

*anton.zeilinger@univie.ac.at

[1] E. Schrödinger, Discussion of probability relations between separated systems, Math. Proc. Cambridge Philos. Soc. 31, 555 (1935).

[2] A. Einstein, B. Podolsky, and N. Rosen, Can quantummechanical description of physical reality be considered complete?, Phys. Rev. 47, 777 (1935).

[3] N. Bohr, Can quantum-mechanical description of physical reality be considered complete?, Phys. Rev. 48, 696 (1935).

[4] J. S. Bell, On the Einstein Podolsky Rosen paradox, Physics 1, 195 (1964).

[5] J.S. Bell, Speakable and Unspeakable in Quantum Mechanics (Cambridge University Press, Cambridge, 1987).

[6] J. F. Clauser, M. A. Horne, A. Shimony, and R. A. Holt, Proposed Experiment to Test Local Hidden-Variable Theories, Phys. Rev. Lett. 23, 880 (1969).

[7] J. F. Clauser and A. Shimony, Bell's theorem. Experimental tests and implications, Rep. Prog. Phys. 41, 1881 (1978).

[8] J.-Å. Larsson, Loopholes in Bell inequality tests of local realism, J. Phys. A 47, 424003 (2014).

[9] N. Brunner, D. Cavalcanti, S. Pironio, V. Scarani, and S. Wehner, Bell nonlocality, Rev. Mod. Phys. 86, 419 (2014).

[10] W. V. Quine, Main trends in recent philosophy: Two dogmas of empiricism, Philos. Rev. 60, 20 (1951).

[11] P. M. Duhem, The Aim and Structure of Physical Theory (Princeton University Press, Princeton, NJ, 1954).

[12] J.-Å. Larsson, M. Giustina, J. Kofler, B. Wittmann, R. Ursin, and S. Ramelow, Bell-inequality violation with entangled photons, free of the coincidence-time loophole, Phys. Rev. A 90, 032107 (2014).

[13] A. Aspect, J. Dalibard, and G. Roger, Experimental Test of Bell's Inequalities Using Time-Varying Analyzers, Phys. Rev. Lett. 49, 1804 (1982).

[14] G. Weihs, T. Jennewein, C. Simon, H. Weinfurter, and A. Zeilinger, Violation of Bell's Inequality under Strict Einstein Locality Conditions, Phys. Rev. Lett. 81, 5039 (1998).

[15] M. A. Rowe, D. Kielpinski, V. Meyer, C. A. Sackett, W. M. Itano, C. Monroe, and D. J. Wineland, Experimental violation of a Bell's inequality with efficient detection, Nature (London) 409, 791 (2001).

[16] M. Giustina, A. Mech, S. Ramelow, B. Wittmann, J. Kofler, J. Beyer, A. Lita, B. Calkins, T. Gerrits, S. W. Nam, R. Ursin, and A. Zeilinger, Bell violation using entangled photons without the fair-sampling assumption, Nature (London) 497, 227 (2013).

[17] B. G. Christensen, K. T. McCusker, J. B. Altepeter, B. Calkins, T. Gerrits, A. E. Lita, A. Miller, L. K. Shalm, Y. Zhang, S. W. Nam, N. Brunner, C. C. W. Lim, N. Gisin, and P. G. Kwiat, 
Detection-Loophole-Free Test of Quantum Nonlocality, and Applications, Phys. Rev. Lett. 111, 130406 (2013).

[18] B. Hensen et al., Loophole-free Bell inequality violation using electron spins separated by 1.3 kilometres, Nature (London) 526, 682 (2015).

[19] M. Giustina et al., Significant-Loophole-Free Test of Bell's Theorem with Entangled Photons, Phys. Rev. Lett. 115, 250401 (2015).

[20] L. K. Shalm et al., Strong Loophole-Free Test of Local Realism, Phys. Rev. Lett. 115, 250402 (2015).

[21] W. Rosenfeld, D. Burchardt, R. Garthoff, K. Redeker, N. Ortegel, M. Rau, and H. Weinfurter, Event-Ready Bell Test Using Entangled Atoms Simultaneously Closing Detection and Locality Loopholes, Phys. Rev. Lett. 119, 010402 (2017).

[22] J. S. Bell, The theory of local beables, Epistemol. Lett. 9, 86 (1976).

[23] A. Shimony, M. A. Horne, and J. F. Clauser, Comment on 'The theory of local beables', Epistemol. Lett. 13, 97 (1976).

[24] J. S. Bell, Free variables and local causality, Epistemol. Lett. 15, 79 (1977).

[25] C.H. Brans, Bell's theorem does not eliminate fully causal hidden variables, Int. J. Theor. Phys. 27, 219 (1988).

[26] J. Kofler, T. Paterek, and Č. Brukner, Experimenter's freedom in Bell's theorem and quantum cryptography, Phys. Rev. A 73, 022104 (2006).

[27] M. J. W. Hall, Local Deterministic Model of Singlet State Correlations Based on Relaxing Measurement Independence, Phys. Rev. Lett. 105, 250404 (2010).

[28] M. J. W. Hall, Relaxed Bell inequalities and KochenSpecker theorems, Phys. Rev. A 84, 022102 (2011).

[29] J. Barrett and N. Gisin, How Much Measurement Independence Is Needed to Demonstrate Nonlocality?, Phys. Rev. Lett. 106, 100406 (2011).

[30] M. Banik, M. Rajjak Gazi, S. Das, A. Rai, and S. Kunkri, Optimal free will on one side in reproducing the singlet correlation, J. Phys. A 45, 205301 (2012).

[31] J. Gallicchio, A. S. Friedman, and D. I. Kaiser, Testing Bell's Inequality with Cosmic Photons: Closing the SettingIndependence Loophole, Phys. Rev. Lett. 112, 110405 (2014).

[32] G. Pütz, D. Rosset, T. J. Barnea, Y.-C. Liang, and N. Gisin, Arbitrarily Small Amount of Measurement Independence Is Sufficient to Manifest Quantum Nonlocality, Phys. Rev. Lett. 113, 190402 (2014).

[33] G. Pütz and N. Gisin, Measurement dependent locality, New J. Phys. 18, 055006 (2016).

[34] M. J. W. Hall, The significance of measurement independence for Bell inequalities and locality, in At the Frontier of Spacetime: Scalar-Tensor Theory, Bell's Inequality, Mach's Principle, Exotic Smoothness, edited by T. Asselmeyer-Maluga (Springer, Switzerland, 2016), Chapter 11, pp. 189-204.

[35] S. Pironio, Random 'choices' and the locality loophole, arXiv:1510.00248.

[36] T. Scheidl, R. Ursin, J. Kofler, S. Ramelow, X.-S. Ma, T. Herbst, L. Ratschbacher, A. Fedrizzi, N. K. Langford, T. Jennewein, and A. Zeilinger, Violation of local realism with freedom of choice, Proc. Natl. Acad. Sci. U.S.A. 107, 19708 (2010).

[37] D. Aktas, S. Tanzilli, A. Martin, G. Pütz, R. Thew, and N. Gisin, Demonstration of Quantum Nonlocality in the Presence of Measurement Dependence, Phys. Rev. Lett. 114, 220404 (2015).

[38] J. Handsteiner et al., Cosmic Bell Test: Measurement Settings from Milky Way Stars, Phys. Rev. Lett. 118, 060401 (2017).

[39] C. Wu, B. Bai, Y. Liu, X. Zhang, M. Yang, Y. Cao, J. Wang, S. Zhang, H. Zhou, X. Shi, X. Ma, J.-G. Ren, J. Zhang, C.-Z. Peng, J. Fan, Q. Zhang, and J.-W. Pan, Random Number Generation with Cosmic Photons, Phys. Rev. Lett. 118, 140402 (2017).

[40] C. Leung, A. Brown, H. Nguyen, A. S. Friedman, D. I. Kaiser, and J. Gallicchio, Astronomical random numbers for quantum foundations experiments, Phys. Rev. A 97, 042120 (2018)

[41] C. Abellán et al. (BIG Bell Test Collaboration), Challenging local realism with human choices, Nature (London) 557, 212 (2018).

[42] One may also consider retrocausal models, in which the relevant hidden variables affect the measurement outcomes from the future [43-45].

[43] O. Costa De Beauregard, S-Matrix, Feynman Zigzag, and Einstein Correlation, Phys. Lett. A 67, 171 (1978).

[44] N. Argaman, Bell's theorem and the causal arrow of time, Am. J. Phys. 78, 1007 (2010).

[45] H. Price and K. Wharton, Disentangling the quantum world, Entropy 17, 7752 (2015).

[46] The Italian Telescopio Nazionale Galileo (TNG) operated on the island of La Palma by the Fundación Galileo Galilei of the INAF (Istituto Nazionale di Astrofisica) at the Spanish Observatorio del Roque de los Muchachos of the Instituto de Astrofisica de Canarias.

[47] The William Herschel Telescope (WHT) operated on the island of La Palma by the Isaac Newton Group of Telescopes (ING) at the Spanish Observatorio del Roque de los Muchachos of the Instituto de Astrofisica de Canarias.

[48] See Supplemental Material at http://link.aps.org/ supplemental/10.1103/PhysRevLett.121.080403, which includes Refs. [49-72], for more information about the experimental setup and data analysis.

[49] P. Sarkar, J. Yadav, B. Pandey, and S. Bharadwaj, The scale of homogeneity of the galaxy distribution in SDSS DR6, Mon. Not. R. Astron. Soc. 399, L128 (2009).

[50] C. Marinoni, J. Bel, and A. Buzzi, The scale of cosmic isotropy, J. Cosmol. Astropart. Phys. 10 (2012) 036.

[51] A. S. Friedman, D. I. Kaiser, and J. Gallicchio, The shared causal pasts and futures of cosmological events, Phys. Rev. D 88, 044038 (2013).

[52] M. G. Mingaliev, Y. V. Sotnikova, R. Y. Udovitskiy, T. V. Mufakharov, E. Nieppola, and A. K. Erkenov, RATAN-600 multi-frequency data for the BL Lacertae objects, Astron. Astrophys. 572, A59 (2014).

[53] W. F. Kern and J. R. Bland, Solid Mensuration with Proofs (Wiley, New York, 1938).

[54] E. Egami, G. Neugebauer, B. T. Soifer, K. Matthews, M. Ressler, E. E. Becklin, T. W. Murphy, Jr., and D. A. Dale, APM $08279+5255$ : Keck near- and mid-infrared highresolution imaging, Astrophys. J. 535, 561 (2000). 
[55] S. Oya, Y. Minowa, H. Terada, M. Watanabe, M. Hattori, Y. Hayano, Y. Saito, M. Ito, T.-S. Pyo, H. Takami, and M. Iye, Spatially resolved near-infrared imaging of a gravitationally lensed quasar, APM $08279+5255$, with adaptive optics on the Subaru telescope, Publ. Astron. Soc. Jpn. 65, 9 (2013).

[56] M. Rauch, The Lyman alpha forest in the spectra of QSOs, Annu. Rev. Astron. Astrophys. 36, 267 (1998).

[57] P. McDonald et al., The Ly $\alpha$ forest power spectrum from the Sloan Digital Sky Survey, Astrophys. J. Suppl. Ser. 163, 80 (2006).

[58] C. R. Benn and S. L. Ellison, Brightness of the night sky over La Palma, New Astron. Rev. 42, 503 (1998).

[59] I. A. B. Meinel, OH emission bands in the spectrum of the night sky, Astrophys. J. 111, 555 (1950).

[60] K. Z. Stanek, T. W.-S. Holoien, C. S. Kochanek, A. B. Davis, G. Simonian, U. Basu, N. Goss, J. F. Beacom, B. J. Shappee, J. L. Prieto, D. Bersier, S. Dong, P. R. Wozniak, J. Brimacombe, D. Szczygiel, and G. Pojmanski, ASAS-SN photometry of QSO BZB J0424+0036, The Astronomer's Telegram $6866 \quad$ (2015), http://www .astronomerstelegram.org/?read=6866.

[61] L. Carrasco, G. Escobedo, A. Porras, E. Recillas, V. Chabushyan, A. Carraminana, and D. Mayya, NIR brightening of the blazar BZB J0424+0036, The Astronomer's Telegram 5712 (2014), http://www.astronomerstelegram .org/?read=5712.

[62] L. Carrasco, A. Porras, E. Recillas, J. Leon-Tavares, V. Chavushyan, and A. Carraminana, An ongoing NIR flare of the QSO BZB J0424+0036, The Astronomer's Telegram 6865 (2015), http://www.astronomerstelegram.org/?read=6865.

[63] L. Carrasco, A. Porras, E. Recillas, J. Leon-Tavares, V. Chavushyan, and A. Carraminana, NIR brightening of the QSO HB89 0422 + 004, The Astronomer's Telegram 6971 (2015), http://www.astronomerstelegram.org/?read=6971.

[64] D. L. King, Atmospheric Extinction at the Roque de los Muchachos Observatory, La Palma, RGO/La Palma Technical Report 31 (1985).

[65] F. G. Saturni, D. Trevese, F. Vagnetti, M. Perna, and M. Dadina, A multi-epoch spectroscopic study of the BAL quasar APM $08279+5255$. II. Emission- and absorptionline variability time lags, Astron. Astrophys. 587, A43 (2016).

[66] B. Sbarufatti, A. Treves, R. Falomo, J. Heidt, J. Kotilainen, and R. Scarpa, ESO very large telescope optical spectroscopy of BL Lacertae objects. II. New redshifts, featureless objects, and classification assessments, Astron. J. 132, 1 (2006).

[67] A. Treves and S. Panzeri, The upward bias in measures of information derived from limited data samples, Neural Comput. 7, 399 (1995).

[68] L. E. Bassham III, A. L. Rukhin, J. Soto, J. R. Nechvatal, M. E. Smid, E. B. Barker, S. D. Leigh, M. Levenson, M. Vangel, D. L. Banks et al., Statistical Test Suite for Random and Pseudorandom Number Generators for Cryptographic Applications, Special Publication (NIST SP) 800-22 Rev 1a (National Institute of Standards and Technology, Gaithersburg, MD, 2010), https://csrc.nist.gov/publications/detail/ sp/800-22/rev-1a/final.
[69] R. D. Gill, Time, Finite Statistics, and Bell's Fifth Position, in Proceedings of Foundations of Probability and Physics2, Math. Modelling in Phys., Engin., and Cogn. Sc., Vol. 5 (Växjö University Press, Sweden, 2003), p. 179, https:// www.eurandom.tue.nl/reports/2003/031-report.pdf.

[70] R. D. Gill, Statistics, causality and Bell's theorem, Stat. Sci. 29, 512 (2014).

[71] P. Bierhorst, A robust mathematical model for a loophole-free Clauser-Horne experiment, J. Phys. A 48, 195302 (2015).

[72] D. Elkouss and S. Wehner, (Nearly) optimal P values for all Bell inequalities, npj Quantum Inf. 2, 16026 (2016).

[73] F. Steinlechner, M. Gilaberte, M. Jofre, T. Scheidl, J. P. Torres, V. Pruneri, and R. Ursin, Efficient heralding of polarization-entangled photons from type- 0 and type-II spontaneous parametric downconversion in periodically poled $\mathrm{KTiOPO}_{4}$, J. Opt. Soc. Am. B 31, 2068 (2014).

[74] T. Kim, M. Fiorentino, and F. N. C. Wong, Phase-Stable Source of Polarization-Entangled Photons Using a Polarization Sagnac Interferometer, in Conference on Lasers and Electro-Optics and 2006 Quantum Electronics and Laser Science Conference, CLEO/QELS 2006, Long Beach, CA, USA, 2006 (IEEE, Long Beach, CA, 2006), pp. 1-5, https:// doi.org/10.1109/CLEO.2006.4628715.

[75] P. J. E. Peebles, Principles of Physical Cosmology (Princeton University Press, Princeton, NJ, 1993).

[76] S. Weinberg, Cosmology (Oxford University Press, Oxford, 2008).

[77] R. D. Blandford and R. Narayan, Cosmological applications of gravitational lensing, Annu. Rev. Astron. Astrophys. 30, 311 (1992).

[78] D. Downes, R. Neri, T. Wiklind, D. J. Wilner, and P. A. Shaver, Detection of CO (4-3), CO (9-8), and dust emission in the broad absorption line quasar APM $08279+5255$ at a redshift of 3.9, Astrophys. J. Lett. 513, L1 (1999).

[79] I. Pâris et al. The Sloan Digital Sky Survey Quasar Catalog: Fourteenth data release, Astron. Astrophys. 613, A51 (2018).

[80] M. S. Shaw, R. W. Romani, G. Cotter, S. E. Healey, P. F. Michelson, A. C. S. Readhead, J. L. Richards, W. Max-Moerbeck, O. G. King, and W. J. Potter, Spectroscopy of the largest ever $\gamma$-ray-selected BL Lac sample, Astrophys. J. 764, 135 (2013).

[81] P. A. R. Ade et al. (Planck Collaboration), Planck 2015 results. XIII. Cosmological parameters, Astron. Astrophys. 594, A13 (2016).

[82] T. Scheidl, R. Ursin, A. Fedrizzi, S. Ramelow, X. S. Ma, T. Herbst, R. Prevedel, L. Ratschbacher, J. Kofler, T. Jennewein, and A. Zeilinger, Feasibility of $300 \mathrm{~km}$ quantum key distribution with entangled states, New J. Phys. 11, 085002 (2009).

[83] B. S. Cirelson, Quantum generalizations of Bell's inequality, Lett. Math. Phys. 4, 93 (1980).

[84] J. Kofler, M. Giustina, J.-Å. Larsson, and M. W. Mitchell, Requirements for a loophole-free photonic Bell test using imperfect setting generators, Phys. Rev. A 93, 032115 (2016).

[85] M. Van Raamsdonk, Lectures on Gravity and Entanglement, in Proceedings, Theoretical Advanced Study Institute in Elementary Particle Physics: New Frontiers in Fields and Strings (TASI 2015): Boulder, CO, USA, 2015 (World 
Scientific, Singapore, 2017), pp. 297-351, https://doi.org/ 10.1142/9789813149441_0005.

[86] M.-H. Li, C. Wu, Y. Zhang, W.-Z. Liu, B. Bai, Y. Liu, W. Zhang, Q. Zhao, H. Li, Z. Wang, L. You, W. J. Munro, J.
Yin, J. Zhang, C.-Z. Peng, X. Ma, Q. Zhang, J. Fan, and J.-W. Pan, following Letter, Test of Local Realism into the Past without Detection and Locality Loopholes, Phys. Rev. Lett. 121, 080404 (2018). 